\title{
Jupiter's aurora in ultraviolet and infrared: Simultaneous observations with the Hubble Space Telescope and the NASA Infrared Telescope Facility
}

\author{
A. Radioti, ${ }^{1}$ M. Lystrup, ${ }^{2}$ B. Bonfond, ${ }^{1}$ D. Grodent,${ }^{1}$ and J.-C. Gérard ${ }^{1}$ \\ Received 31 January 2013; revised 8 March 2013; accepted 25 March 2013.
}

[1] We compare Jupiter's northern auroral emissions in infrared (IR) and ultraviolet (UV) using ground-based IR observations from the NASA Infrared Telescope Facility and UV observations from Hubble Space Telescope on 16 December 2000, the only date for which simultaneous observations in the two wavelength regions exist. We use polar projections and longitudinal brightness cuts to compare the IR $\left(\mathrm{H}_{3}^{+}\right.$ions $)$and $\mathrm{UV}\left(\mathrm{H}_{2}, \mathrm{H}\right.$, and Lyman-alpha) aurorae, consisting of the main auroral emission, emission regions both poleward and equatorward of the main emission, and those associated with the Io footprint and its extended tail. We demonstrate that (1) the IR main emission and the equatorward diffuse emissions are generally good proxies for the UV and vice versa, (2) the spatial distribution and temporal behavior of UV and IR emissions within the main emission, at high magnetic latitudes, differ substantially, (3) UV and IR emissions associated with the Io interaction appear at the Io footprint and along an extended (downstream) tail but differ in relative brightness. While the UV aurora is excited directly, the IR aurora is a thermal emission, its intensity depends on both the number density of the $\mathrm{H}_{3}^{+}$ions and the temperature. Three main factors may contribute to the observed morphological differences of the simultaneous emissions in the two wavelengths, namely ion transport, local heating, and the energy of the precipitating electrons. We estimate the $\mathrm{H}_{3}^{+}$ion transport distances, based on the ion lifetime and suggest that ion transport cannot account for large-scale morphological differences between the UV and IR emissions. We propose that neutral gas heating by particle precipitation and Joule heating locally enhances the $\mathrm{H}_{3}^{+}$emission with no UV counterpart. Additionally, we estimate that local temperature variations are reflected in the IR emission with a time lag of several hours with respect to the UV. Finally, high precipitating electron energies exceeding a certain value might lead to chemical loss of the low altitude $\mathrm{H}_{3}^{+}$ions, suppress the lower IR emitting layers, and contribute to the observed differences of the emissions between the two wavelength regimes.

Citation: Radioti, A., M. Lystrup, B. Bonfond, D. Grodent, and J.-C. Gérard (2013), Jupiter's aurora in ultraviolet and infrared: Simultaneous observations with the Hubble Space Telescope and the NASA Infrared Telescope Facility, J. Geophys. Res. Space Physics, 118, doi:10.1002/jgra.50245.

\section{Introduction}

[2] Auroral emissions at Jupiter have been observed at wavelengths in the radio, ultraviolet (UV), visible, infrared (IR), and X-ray wavelengths. Studies in the UV and IR have

\footnotetext{
${ }^{1}$ Laboratoire de Physique Atmosphérique et Planétaire, Institut d'Astrophysique et de Géophysique, Université de Liège, Liège, Belgium.

${ }^{2}$ School of Physics, Astronomy, and Computational Sciences, George Mason University, Fairfax, Virginia, USA.

Corresponding author: A. Radioti, Laboratoire de Physique Atmosphérique et Planétaire, Institut d'Astrophysique et de Géophysique, Université de Liège, 4000 Liège, Belgium. (a.radioti@ulg.ac.be)

(C)2013. American Geophysical Union. All Rights Reserved. 2169-9380/13/10.1002/jgra.50245
}

been particularly fruitful, the former in revealing the complex morphology and variability of the aurora and the latter in showing the upper atmosphere's response to magnetospheric processes. Jupiter's UV aurora, in the $80-165 \mathrm{~nm}$ wavelength region, results from inelastic collisions of energetic electrons of magnetospheric origin with atmospheric molecular hydrogen $\left(\mathrm{H}_{2}, \mathrm{H}\right.$, and Lyman-alpha). Jupiter's IR aurora is due primarily to thermal emissions from the $\mathrm{H}_{3}^{+}$ molecular ion at altitudes above the Jovian homopause. At these high altitudes in the auroral/polar latitudes, $\mathrm{H}_{2}$ is ionized by energetic electrons $\left(\mathrm{e}^{*}\right)$ that are precipitated into the upper atmosphere as a result of magnetospheric processes $\mathrm{H}_{2}+\mathrm{e}^{*} \rightarrow \mathrm{H}_{2}^{+}+\mathrm{e}+\mathrm{e} . \mathrm{H}_{3}^{+}$ions are subsequently produced copiously and rapidly via $\mathrm{H}_{2}+\mathrm{H}_{2}^{+} \rightarrow \mathrm{H}_{3}^{+}+\mathrm{H}$. Observation of $\mathrm{H}_{3}^{+} \mathrm{IR}$ emissions is a good probe of the upper 
atmosphere, as $\mathrm{H}_{3}^{+}$is efficiently destroyed by hydrocarbons $\left(\mathrm{H}_{3}^{+}+\mathrm{X} \rightarrow \mathrm{H}_{2}+\mathrm{HX}^{+}\right)$, thus eliminating $\mathrm{H}_{3}^{+}$below the homopause [Connerney and Satoh, 2000].

[3] Whereas the UV auroral emissions, which originate from lower altitudes $(250 \mathrm{~km})$ [Vasavada et al., 1999] than the IR $(1000 \mathrm{~km})$ [Lystrup et al., 2008], are a good tracer of instantaneous energy inputs, the IR aurora reflects how the atmosphere responds to those inputs. However, the only comparative study at Jupiter to date is a brief discussion by Clarke et al. [2004]. In the present work, we take advantage of simultaneous observations of Jupiter's aurora made in the UV by the Hubble Space Telescope (HST) and the near-IR by the NASA Infrared Telescope Facility (IRTF) on 16 December 2000. In this study, we are concerned with the comparative morphology of Jupiter's aurora in these two wavelength regimes. The general morphology of the Jovian aurora can be described in terms of four components, namely the emissions belonging to the main auroral emission, those poleward and equatorward of the main emission, and those related to the satellite interactions with the Jovian magnetosphere.

[4] The main emission is generally associated with upward field-aligned currents driven by the breakdown of corotation between the planet and the vast corotating plasma sheet, which is initially supplied as neutral gas by volcanic activity of Jupiter's moon Io. Theoretical work suggests that corotation breakdown occurring at equatorial magnetospheric distances $\sim 15-40 R_{J}$ generates a global current system whose upward branch is associated with auroral precipitation [Cowley and Bunce, 2001; Hill, 2001]. Jupiter's main auroral emission in the UV forms a relative stable strip of emission around the magnetic pole with variability on time scales of minutes to hours [Gérard et al., 1994; Ballester, 1996; Grodent et al., 2003a]. This is estimated to contribute $75 \%$ of the Jovian auroral brightness integrated over high latitudes [Nichols et al., 2009b], and its brightness has been shown to vary between 50 and $500 \mathrm{kR}$ [Grodent et al., 2003a]. HST observations of the dayside aurora have shown that there is a persistent asymmetry between the dawnside main emission, which is most of the time narrow and continuous, and the duskside main emission, which is usually broader and sometimes appears to separate into several arcs. Additionally, its shape may be influenced by a localized magnetic anomaly, a feature fixed in the SIII polar coordinate system [Grodent et al., 2008]. In UV images, discontinuities along the main emission where emissions fall to very low levels have been interpreted as locations of possible downward field-aligned currents [Radioti et al., 2008a]. Satoh and Connerney [1999] demonstrated that the most intense IR emissions are found in the auroral zone which magnetically maps to $12-30 R_{J}$, thus corresponding approximately to the main UV emission. IR spectroscopic observations showed that the main auroral oval is dominated by electrojet (ionospheric plasma flow), flowing with velocities in the line of sight between $0.5 \mathrm{~km} \mathrm{~s}^{-1}$ and $1.5 \mathrm{~km} \mathrm{~s}^{-1}$ in the frame of reference corotating with the planet [Rego, 1999; Stallard et al., 2001]. This flows in a clockwise direction, viewed from the northern rotational pole, in a counter-rotational sense. The mechanism for accelerating these ions directly stems from corotation breakdown of magnetospheric plasma [Cowley and Bunce, 2001]. Such studies have also revealed that the $\mathrm{H}_{3}^{+}$temperature in the region of the main emission is in the range $1000-1200 \mathrm{~K}$ [Lystrup et al., 2008; Stallard et al., 2002; Raynaud et al., 2004], with column densities in the range $3-4 \times 10^{12} \mathrm{~cm}^{-2}$ [Lystrup et al., 2008].

[5] Poleward of the main emission, the Jovian aurora is highly spatially and temporally variable. It has been suggested that the polar emissions are magnetically connected to the outer magnetosphere and possibly related to a sector of the Dungey and Vasyliunas cycle flows [Cowley et al., 2003; Grodent et al., 2003b]. UV auroral observations have shown the occasional appearance of parallel arc structures and nightside spots located poleward of the main emission in the dawn and nightside sector, which have been suggested to be associated with magnetotail reconnection [Grodent et al., 2003a, 2004; Radioti et al., 2008b]. Additionally, quasi sunaligned polar auroral filaments have been detected spanning the highly variable region poleward of the main oval, implying a relation to tail dynamics [Nichols et al., 2009a]. Finally, UV data revealed the presence of bright isolated transient features located poleward of the main oval such as transient flares, which are possibly related to pulsed reconnection at the dayside magnetopause [Waite et al., 2001; Bunce et al., 2004; Bonfond et al., 2011]. Polar emissions have also been observed at IR wavelengths, and it has been shown that $\sim 45 \%$ of the IR power emitted from the entire auroral region originates from the polar zone [Satoh and Connerney, 1999]. Recently, IR nightside spots have been associated with magnetotail reconnection [Radioti et al., 2011]. Three main regions poleward of the main emission are noted [Stallard et al., 2001, 2003]: the first two are the rotating and fixed dark polar region which appear to be strongly red shifted relative to the planet, with velocity profiles in the line of sight up to $-2.5 \mathrm{~km} \mathrm{~s}^{-1}$ in the frame of reference corotating with the planet, flowing in a clockwise direction, viewed from the north, in a counter-rotational sense. The third one is the bright polar region, which is observed to have weakly blue-shifted emission relative to the planet. The dark polar region is defined as the area between the main emission and patchy polar aurora of consistently very low emission in the UV but relatively brighter in the IR. The ion flow velocities observed for the fixed dark polar region indicate a nearstagnant plasma in the frame of reference fixed with respect to magnetic pole and therefore suggested to be connected to open magnetotail field lines [Stallard et al., 2003].

[6] Equatorward of the main emission but poleward of the Io footprint, diffuse emissions have been observed in both the UV and the IR. The UV equatorward diffuse emissions extend from the main emission toward lower latitudes, occasionally forming a discrete belt of emissions parallel to the main emission and/or patchy irregular emissions [Grodent et al., 2003a]. Radioti et al. [2009] suggested that part of the equatorward UV diffuse emission is associated with electron scattering into the loss cone by whistler mode waves at the pitch angle distribution boundary, leading to electron precipitation in the ionosphere. The pitch angle distribution boundary is defined as a transition region of the electron pitch angle distribution from pancake to bidirectional, a region regularly observed between 10 and 17 $R_{J}$ [Tomás et al., 2004]. In the auroral region between the main emission and Io footprint, UV and IR transient auroral patches are occasionally observed and have been related to magnetospheric injections [Mauk et al., 2002; Bonfond 
et al., 2012]. IR emissions observed in a zone corresponding to $8-12 R_{J}$ (a region which approximately co-locates with the equatorward diffuse emission region) have been shown to contribute $\sim 20 \%$ of the total IR power emitted from the entire region [Satoh and Connerney, 1999].

[7] Finally, electromagnetic interactions among Jupiter and its moons result in auroral signatures equatorward of the main emission, most prominently the Jupiter-Io interaction, at magnetospheric distances $\sim 5.9 R_{J}$. Evidence of this interaction has been observed in the form of the Io footprint in the IR [Connerney et al., 1993] and UV [Clarke et al., 1996]. Observations of the Io footprint have revealed a complex morphological structure comprising at least three individual spots and an extended tail [Bonfond, 2012; Connerney and Satoh, 2000]. While the brightest spot is associated with the direct propagation of Alfvén waves out of the Io plasma torus, the two other spots are associated with electrons being accelerated in one hemisphere and precipitating in the opposite one and to reflection of the Alfvén waves on the plasma torus density gradient [Neubauer et al., 1980; Gurnett and Goertz et al., 1981]. The extended tail could be related either to the acceleration of stagnant plasma at Io [e.g., Hill and Vasyliûnas, 2002] or to subsequent reflections of Alfvén waves. Footprint emissions from Europa and Ganymede have also been observed in the UV [Clarke et al., 2002; Grodent et al., 2006, 2009], but so far not in the IR.

\section{IR and UV Data}

[8] Between 21 July 1995 and 19 December 2000, Connerney and Satoh conducted a campaign of imaging observations of Jupiter's IR aurora using the NASA IR Telescope Facility on Mauna Kea in Hawaii. This long-term monitoring program used the now-defunct NSFCAM imager [Shure et al., 1994], a 1-5 $\mu \mathrm{m}$ imager with a $256 \times 256$ pixel array and pixel scale of $0.148^{\prime \prime}$, with a $0.04 \mu \mathrm{m}$ wide filter centered at $3.43 \mu \mathrm{m}$. Observations at $3.4 \mu \mathrm{m}$ have been selected because (i) this region is within the Earth's atmospheric $L$-window, (ii) they collect several intense $\mathrm{H}_{3}^{+}$lines [Kao et al., 1991], and (iii) strong methane absorption at this wavelength effectively masks all thermal emissions and reflected sunlight from deeper levels in the atmosphere. As a result, the $\mathrm{H}_{3}^{+}$aurora at $3.4 \mu \mathrm{m}$ stands up with high contrast against the dark planetary disk. Over the total of 51 nights of observations, 8808 images were recorded and were subsequently reduced and processed as described in Satoh and Connerney [1999]. Maps and polar projections were created using methods previously employed for UV images from the Hubble Space Telescope by the Liège group. The auroral IR emission brightness reflects both the column density of $\mathrm{H}_{3}^{+}$ ions and the $\mathrm{H}_{3}^{+}$rotational temperature. The intensity of a given rotational line is thus proportional to the $\mathrm{H}_{3}^{+}$density integrated along the line of sight and depends on temperature through the Boltzmann population on each excited rotational level. In this study, the measured IR intensity refers to the set of lines within the $0.04 \mu \mathrm{m}$ filter passband belonging to the fundamental transition. The IR image analyzed in this work was taken at 12:20 UT on 16 December 2000 and is compared with the simultaneous UV observations.

[9] The UV image used in this study was obtained from the Hubble Space Telescope's photon-counting detector Multi-Anode Micro Channels Array (MAMA) of the Space
Telescope Imaging Spectrograph (STIS). The MAMA array consists of $1024 \times 1024$ pixels providing a field of view (FOV) of $24.7^{\prime \prime} \times 24.7^{\prime \prime}$ with an $\sim 0.08^{\prime \prime}$ full width at half maximum point spread function. The instrument was used in clear mode covering a spatial band of 115-170 nm. The emission includes the $\mathrm{H}$ Lyman-alpha line and $\mathrm{H}_{2}$ bands from the Lyman and Werner systems. The observed counts in the filter bandwidth have been converted into $\mathrm{kR}$ for the full $\mathrm{H}_{2}$ UV bandwidth (700-1800 A) with conversion factors determined by Gustin et al. [2012] assuming a color ratio of 2.5, a typical value representing the hydrocarbon absorption. This conversion assumes that Lyman alpha contributes $9.1 \%$ of the total unabsorbed auroral signal. The starting time of the image was at 12:19 UT on 16 December with an exposure time of $110 \mathrm{~s}$.

\section{Comparison of the IR and UV Aurora}

[10] Simultaneous IR and UV observations are rare. There are other dates for which IR and UV observations were recorded close in time, for example, as presented in Radioti et al. [2011] for observations on 26 July 1998, but only on 16 December 2000 were simultaneous observations recorded. Therefore, in this study we concentrate on images on this latter date.

\subsection{Polar Projections}

[11] We have produced polar projections of the UV and IR images, assuming emission altitudes of $250 \mathrm{~km}$ [Vasavada et al., 1999] and $1000 \mathrm{~km}$ [Lystrup et al., 2008], respectively, above the 1 bar reference altitude. Grodent et al. [2003b] computed the mapping error of HST observations of the Jovian aurora, assuming a pointing uncertainty of 4 pixels. Even though this error significantly increases close to the limb, it leads to an uncertainty of only $\sim 1^{\circ}$ in longitude/latitude for the regions of interest in the present study. The pixel size of IRTF images is 6 times larger than for the STIS images, and we estimate the pointing accuracy to be around 2 pixels, similar to that reported by Satoh and Connerney [1999]. As a consequence, the uncertainty reaches $3^{\circ}$ in the regions of interest. A realistic uncertainty of $200 \mathrm{~km}$ in the emission altitude would correspond roughly to 2 pixels and third of a pixel for UV and IR images, respectively, which is thus small compared to the pointing uncertainty.

[12] Figure 1 shows the UV and IR polar projections as well as the different morphological regions under study (Io footprint, equatorward diffuse emission, main emission, and polar emission). In a first view, the simultaneous UV and IR emissions appear to have several features in common, such as the existence of the Io footprint, a quite well defined main emission, a broad equatorward diffuse emission region in the dusk sector, and randomly distributed polar emission. However, comparing the emissions in greater detail, one can identify several morphological differences at the two wavelengths: (i) the relative intensity of the main emission as a function of local time does not seem to vary in the same way in the UV and IR, (ii) the polar emissions are not always co-located and their sizes differ dramatically at the two wavelengths, and (iii) the Io footprint in this pair of images is less evident in the IR than it is in the UV. 


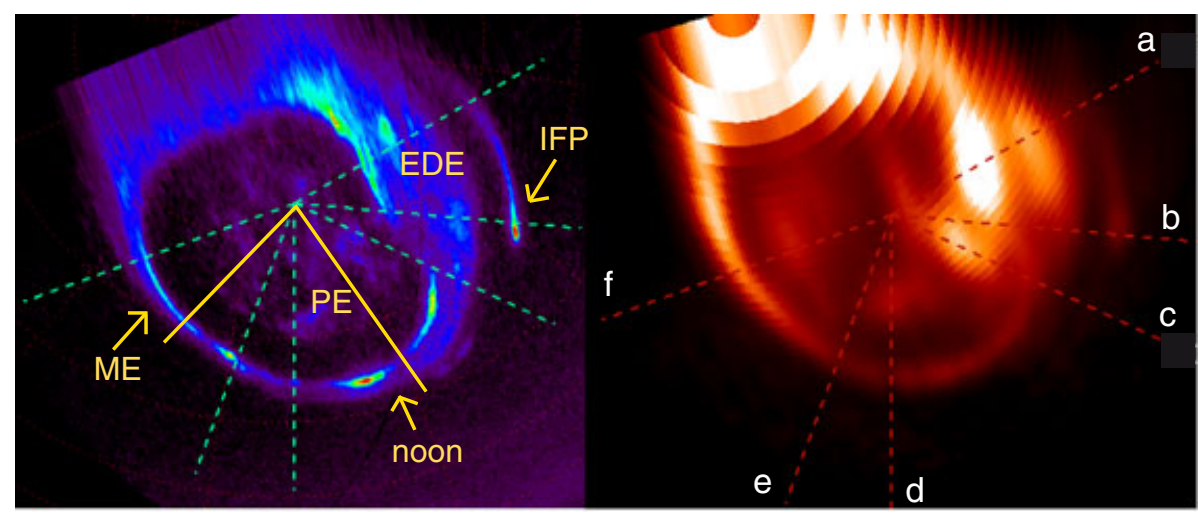

Figure 1. Polar projections of the auroral images in the UV from the Hubble Space Telescope (left) and the IR from the IRTF (right) showing the aurora on the two different wavelengths observed simultaneously on 16 December 2000 at 12:20 UT. The dashed lines show the cuts that were used to create relative brightness profiles shown in Figure2. The yellow solid lines in the UV projection show the sector of the main emission analyzed separately in Figure4. The main features under study are indicated on the UV projection: main emission (ME), polar emission (PE), equatorward diffuse emission (EDE), and Io footprint (IFP). Both images are at CML $159^{\circ}$ and are presented in System III longitude with $180^{\circ}$ downward.

\subsection{Latitudinal Cuts}

[13] In order to study the morphological similarities and differences of the auroral emissions in the two simultaneous data sets, we present a detailed comparison of the different auroral features along latitudinal cuts with respect to the morphological center of the main UV emission (latitude $+74^{\circ} \mathrm{N}$, SIII longitude $185^{\circ}$, as estimated by Grodent et al. [2004]). We create brightness versus pixel profiles for six locations of interest away from the limb in order to minimize residual limb brightening effects in the IR image. One pixel corresponds approximately to one fourth of a degree in longitude and latitude. The latitudinal cuts from a to $f$, indicated on the UV and IR projections in Figure 1 are chosen to cross the different aspects of the various morphological features mentioned above. In order to take into account the difference in spatial resolution between the IR and UV images due to the inherent differences in the instruments and in observing conditions, we have smoothed the UV image so that the pixel size in the UV image corresponds to the pixel size of the IR image (pixel size of IR IRTF is 6 times larger than UV STIS). Figure 2 displays the normalized to the peak in the main emission brightness profile of the aurora, as a function of angular distance measured from the morphological center of the main emission for profile a. Figure $2 \mathrm{a}$ shows the IR (red) and raw UV (blue) profiles, while Figure 2 b shows the IR (red) and smoothed UV (blue) profiles along the same cut. In the following, we use the smoothed UV profiles for the direct comparison with IR. Figure 3 displays the normalized to the peak in the main emission brightness profiles, as a function of angular distance measured from the morphological center of the main emission for the UV (blue left axis) and IR (red right axis). The main emission, which is relatively stable in time and easy to identify is chosen to be the reference emission for the normalized profiles.

[14] Profile a is a cut extending from the center of the aurora through a patch of relatively weak polar auroral emissions at about pixel 20 , then passes through the duskside main emission with a peak brightness near pixel 70 , through the equatorward diffuse emission that extends from pixels $\sim 80$ to 130 , and finally through the tail of the UV Io footprint at about pixel 175. The brightness of the polar emission patch is about $45 \%$ that of the peak emission in the main

a)

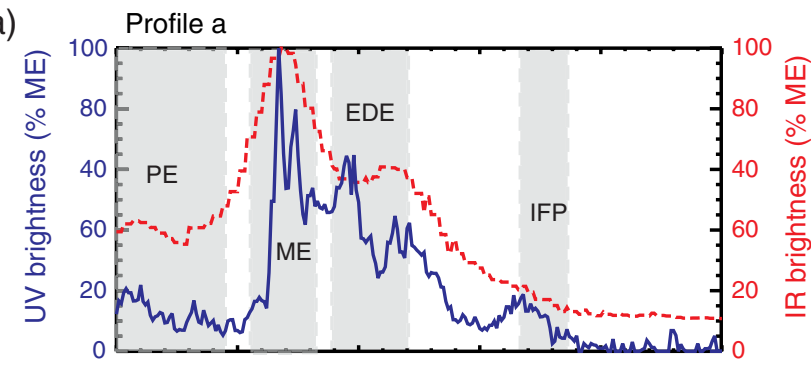

b)

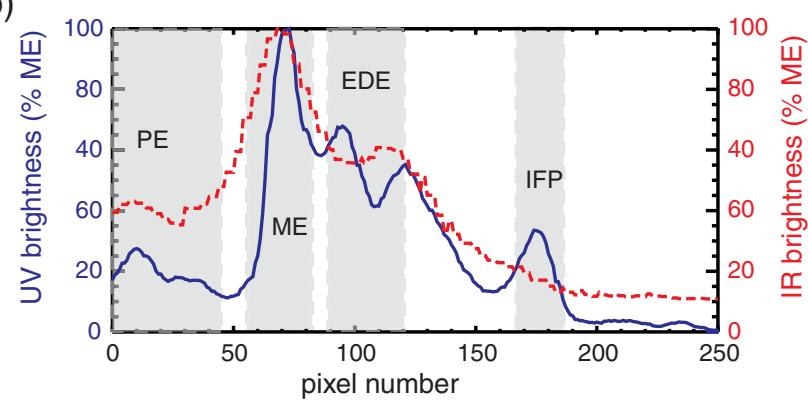

Figure 2. Brightness profiles of the UV (blue) and IR (red) auroral emissions (above the background emission) as a function of angular distance measured from the morphological center of the main emission (latitude $+74^{\circ}$, SIII longitude $185^{\circ}$ ) for profile a (position shown in Figure 1). The $y$ axes are brightness values in terms of percentage of the main emission brightness in UV (left axis) and IR (right axis). The UV profile is shown (a) unsmoothed and (b) smoothed for direct comparison with the IR. The main emission (ME), polar emission (PE), equatorward diffuse emission (EDE), Io footprint (IFP), and dark polar region (DPR) auroral features are shaded in gray. 

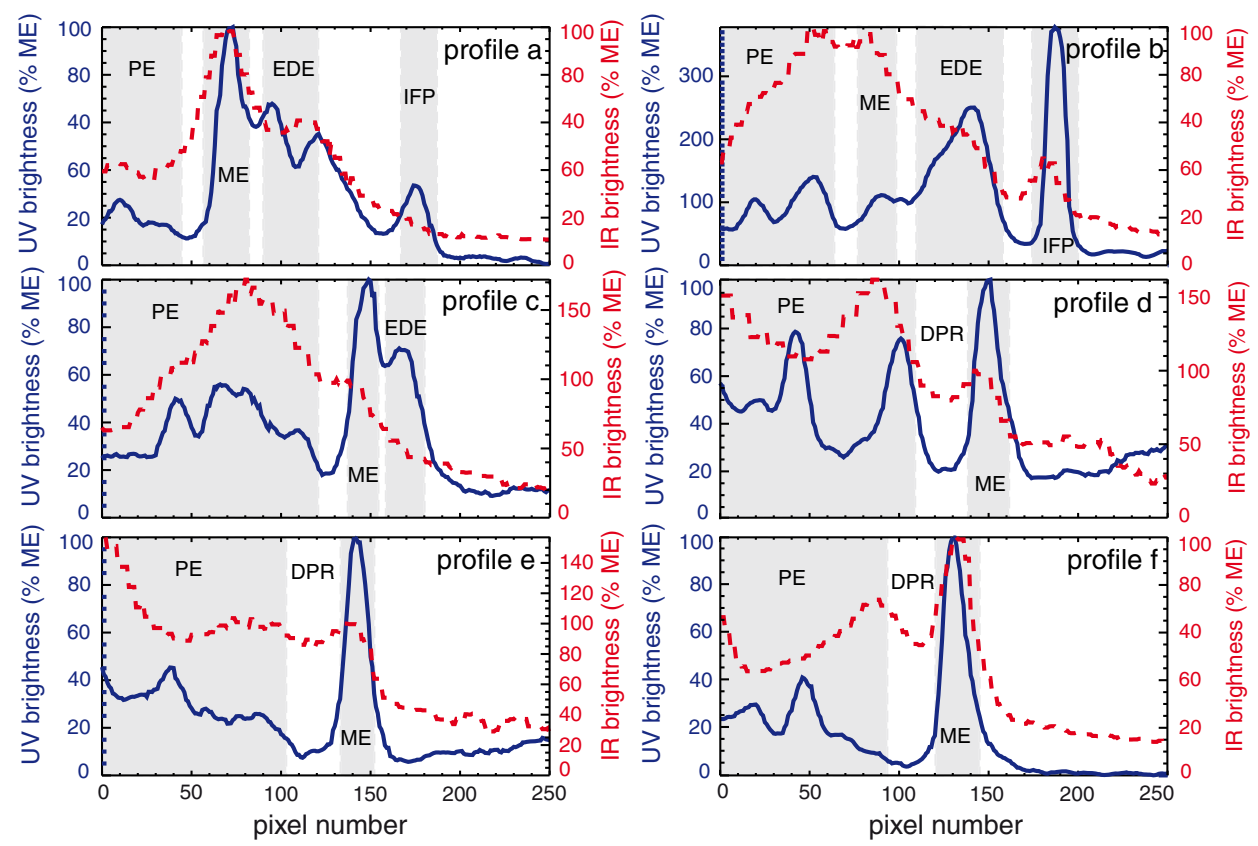

Figure 3. Brightness profiles of the smoothed UV and IR auroral emissions (above the background emission) as a function of angular distance measured from the morphological center of the main emission (latitude $+74^{\circ}$, SIII longitude $185^{\circ}$ ). Profile labels correspond to positions shown in Figure 1. The same format as Figure 2.

emission for the IR and about $30 \%$ for the UV. The brightness of the Io tail in the UV reaches nearly $35 \%$ that of the main emission, but in the IR, a peak associated with the feature is more difficult to identify, though it is evident in the image (Figure 1). The equatorward diffuse emission along this profile consists of two separate peaks in the UV, while such a morphology is not present in the IR. In general, this profile shows the best correspondence between the UV and the IR emissions.

[15] Profile b extends from the center of the aurora through polar emissions and through the main emission with peak brightness centered at pixel 90, through equatorward diffuse emission between pixels 110 and 160 (its identification is based also on the principal image in Figure 1), and through the main spot of the Io footprint at about pixel 190. The UV main emission region is relatively low in brightness, while the IR main emission is the brightest feature along this profile. In the UV, the brightness of the primary Io footprint spot exceeds that of the brightest part of the main emission by more than $250 \%$, whereas in the IR, the Io footprint brightness is only about $40 \%$ the main emission brightness, although there is not a large difference in the spatial extent of the Io footprint. In the UV, one can hardly see a bright polar emission, while in IR, the cut crosses an extended bright region.

[16] Profile c extends from the center of the aurora through a polar auroral patch, through the main emission at pixels $140-160$, and through equatorward diffuse emission. The bright polar patch in the IR is the same one as in the cut along profile $b$ and is more confined in size in the UV compared to IR. The UV brightness of the polar emission is half of that of the main emission, while in the IR, the polar emission is about $51 \%$ brighter than the main emission. Whereas the UV and IR equatorward diffuse emission are co-located for profiles $\mathrm{a}$ and $\mathrm{b}$, along profile $\mathrm{c}$ there appears to be no IR counterpart of this auroral feature.

[17] Profile $d$ extends from the center of the aurora through a bright patch of polar emission, and the main emission at pixels roughly $140-160$. As in the case of profile c, the IR brightness of the bright polar emission is greater than the main emission, about $160 \%$ that of the main emission, whereas the UV polar emission is about $80 \%$ the brightness of the main emission. It is also seen that at locations between the main emission and the bright polar patch, the UV brightness falls to its lowest values, while the IR emission remains about $50 \%$ of the brightness of the bright polar spot (polar emission) and about $80 \%$ of the brightness of the main emission. This location corresponds to part of the dark polar region. Equatorward diffuse emission is not observed either in the IR or in the UV in this sector.

[18] Profiles e and f extend from the center of the aurora, through polar aurora, through the dark polar region centered at roughly pixel 120 (profile e) and 110 (profile f), and through the main emission near pixel 140 (profile e) and 130 (profile f). Here again, the peaks in the UV and IR main emissions are co-located, and the equatorward diffuse emission is not observed at all and the dark polar region intensity drops to near zero in the UV, while it remains quite bright $(50-80 \%$ that of the main emission) in the IR.

[19] Figure 4 shows the brightness profile of the peak values of the main emission in $\mathrm{kR}$ (above the background emission), as a function of the meridional longitude (degrees) with respect to the morphological center of the main emission and SIII longitude for the UV (blue) and IR (red). The UV profile around $165^{\circ}$ SIII longitude exhibits a remarkable peak, while is not the case for the IR profile. Between $170^{\circ}$ and $188^{\circ}$ (SIII), the UV brightness along the profile reaches low values of $\sim 100 \mathrm{kR}$. This region, which 


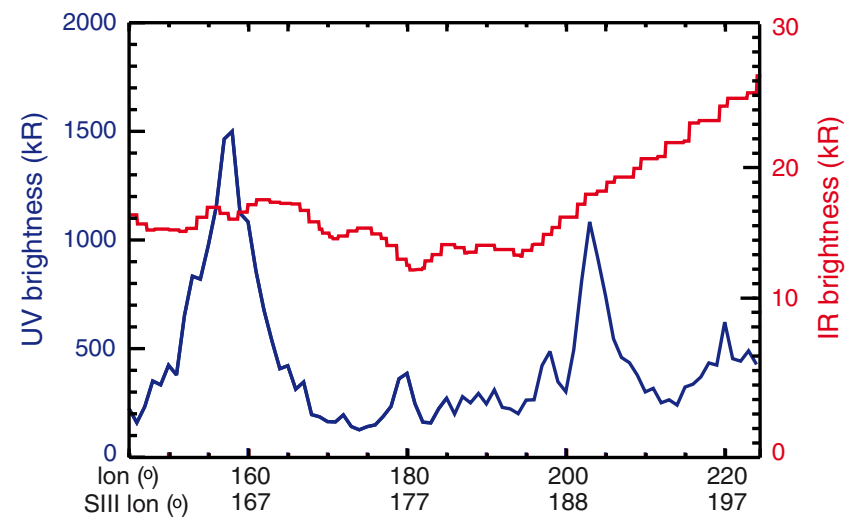

Figure 4. Brightness profiles along the peak values of main emission (in $\mathrm{kR}$, above the background emission) in UV (blue) and IR (red), as a function of the longitude (degree) of meridians with respect to the morphological center of the main emission and in SIII longitude. The profiles demonstrate the brightness variations along the main emission for the two wavelengths simultaneously for the sector between the solid yellow lines in the UV projection in Figure 1.

corresponds to pre-noon local time sector, is defined in previous studies as the "discontinuity region" [Radioti et al., 2008a], a region fixed in local time (statistically it maps to 9-13 LT), which corresponds to downward field-aligned currents and therefore to dim UV emission. The brightness of IR main emission along the profile varies with local time. Between $170^{\circ}$ and $188^{\circ}$ (SIII), the region where the UV discontinuity is observed, the IR brightness slightly decreases but this drop is not as remarkable as the one in the UV. Finally, between $185^{\circ}$ and $200^{\circ}$ (SIII), the IR brightness increases with longitude while the UV after a well-defined peak in the same region drops significantly. All these point to the fact that the UV and IR profiles along the main emission vary independently of each other.

\section{Discussion}

\subsection{Auroral Electron Energy}

[20] In order to discuss and explain the above observations, we need to briefly introduce the electron energies associated with the respective auroral regions as well as their observed altitudes. The altitude of the UV aurora decreases monotonically with the mean energy of the auroral electrons. The altitude of IR aurora also drops with increasing energy. The peak of the IR emission is expected to be located above that of the FUV aurora and near $10 \mathrm{keV}$ [Tao et al., 2011]. For a given precipitation electron flux, the IR brightness decreases as the precipitation hardens and thus penetrates into the hydrocarbon layer where the $\mathrm{H}_{3}^{+}$ions are quickly lost. Based on mean electron energy value determinations from the FUV color ratio, the main emission appears to be excited by precipitation in the range from 10 to several hundred $\mathrm{keV}$ [Gustin et al., 2004]. Its location is similar to the visible $\mathrm{H}_{2}$ emission whose maximum was found near $250 \mathrm{~km}$ [Vasavada et al., 1999]. The emissions poleward of the main emission are excited by electron population in the energy range of 40-120 keV [Gérard et al., 2003], but its altitude has not been directly determined.
The altitude of the FUV Io footprints is about $900 \mathrm{~km}$ [Bonfond, 2010], indicating softer characteristic electron energies than the other auroral components, which are located in lower altitudes.

\subsection{Transport of $\mathrm{H}_{3}^{+}$Ions}

[21] We now examine whether transport of ionospheric ions and local heating may also play a role in interpreting the observed differences between the localization of some UV and IR features. $\mathrm{H}_{3}^{+}$ions are lost through recombination with ambient thermal electrons followed by dissociative recombination. The effective lifetime of a $\mathrm{H}_{3}^{+}$ion is given by $\tau=1 /(\alpha$ $\mathrm{N}_{e}$ ), where $\alpha$ is the recombination coefficient approximately equal to $2.3 \times 10^{-7} \mathrm{~cm}^{3} \mathrm{~s}^{-1}$ at $300 \mathrm{~K}$. In the auroral region, the electron density $\mathrm{N}_{e}$ itself depends on the characteristics (number flux, energy spectrum) of the incident auroral electrons. $\mathrm{H}_{3}^{+}$ion density profiles have been calculated with 1-D [Tao et al., 2011] and 3-D models [Bougher et al., 2005] and the peak electron densities range between $1 \times 10^{5}$ and $1 \times 10^{6}$ $\mathrm{cm}^{-3}$. The corresponding $\mathrm{H}_{3}^{+}$chemical lifetime thus ranges between 4 and $40 \mathrm{~s}$. Combining a more likely lifetime of $4 \mathrm{~s}$ with the velocities on the order of $10-50 \mathrm{~m} \mathrm{~s}^{-1}$ in the corotation frame calculated by Achilleos et al. [2001], the ions may be carried over a distance of only a few tens of meters in the collisional region. Therefore, we conclude that horizontal transport of $\mathrm{H}_{3}^{+}$ions is expected to play a minor role and cannot explain morphological differences between the ultraviolet and the IR auroral emission. However, measurements of the Doppler shift of $\mathrm{H}_{3}^{+}$IR emission have revealed that the main auroral emission region is dominated by electrojet (ionospheric plasma flow), flowing at velocities in the line of sight between $0.5 \mathrm{~km} \mathrm{~s}^{-1}$ and $1.5 \mathrm{~km} \mathrm{~s}^{-1}$ in the frame of reference corotating with the planet [Rego et al., 1999; Stallard et al., 2001], much larger than the modeled velocities estimated by Achilleos et al. [2001]. In the IR dark polar region, ion velocities in the line of sight up to $2.5 \mathrm{~km} \mathrm{~s}^{-1}$ in the frame of reference corotating with the planet. Repeating our estimation of the transport distance, $\mathrm{H}_{3}^{+}$ions can move about 5 to $10 \mathrm{~km}$ along the main oval or across the polar region. Depending on the ionization rate, the transport could vary, since the electron density, and thus the effective $\mathrm{H}_{3}^{+}$ lifetime, varies approximately with the square root of the ionization rate which is proportional to the electron energy flux. Weaker auroral precipitation (for example, $\sim 100$ times weaker) would result in longer $\mathrm{H}_{3}^{+}$chemical lifetime $(\sim 10$ times longer) and thus larger transport distance $(\sim 100 \mathrm{~km})$. We note that these values are still small in comparison to the size of the auroral features exhibiting differences between the UV and the IR.

\subsection{Local Heating}

[22] A third, more likely, possible cause of morphological differences is local heating affecting the gas temperature and thus the brightness of the $\mathrm{H}_{3}^{+}$IR emission. The intensity of a transition is proportional to $\exp (-E / k T)$, where $E$ is the energy of the upper level $\left(=2882 \mathrm{~cm}^{-1}\right), k$ the Boltzmann constant, and $T$ the temperature. For example, a temperature increase from 800 to $1200 \mathrm{~K}$ produces an intensity increase by factor 5.3, a large value able to substantially affect the brightness distribution. Accounting for nonlocal thermodynamic equilibrium effects, Tao et al. [2011] obtained a slightly less factor (about 4). We note that local heating may 
be caused by direct electron precipitation, Joule heating, and compressional heating in regions of converging winds. The importance of Joule heating in the energy balance of the Jovian thermosphere was discussed in detail by Bougher et al. [2005]. They showed that it is by far the main source of heating in the upper atmosphere.

[23] Local temperature changes are controlled by balance between heating and cooling terms, including the transport contribution. A time lag exists between instantaneous particle precipitation changes, directly reflected by the UV $\mathrm{H}_{2}$ emission and the IR atmospheric thermal response. A crude estimate is given by the characteristic time for $\mathrm{H}_{3}^{+}$ cooling, the dominant heat loss term near the $\mathrm{H}_{3}^{+}$emission peak. Using a cooling rate of $2 \times 10^{5} \mathrm{eV} \mathrm{cm}^{-3} \mathrm{~s}^{-1}$ at $500 \mathrm{~km}$ and a corresponding $\mathrm{H}_{2}$ number density of $3 \times 10^{11} \mathrm{~cm}^{-3}$ [Grodent et al., 2001], the time for a $10 \mathrm{~K}$ temperature drop is on the order of $10 \mathrm{~h}$. That means that while temperature variations are directly reflected by the UV emissions, they are consistently delayed in IR thermal emissions.

\subsection{Main Emission: UV-IR Morphological Comparison}

[24] The present observations indicate that the UV main emission is generally a good proxy for the IR and vice versa. This is not surprising since the main emission is not a transient feature but rather a stable one. However, looking closely in the brightness variations along the main emission (Figure 4), one concludes that both brightness profiles in UV and IR vary independently of each other. Increases and decreases in brightness along the main emission are not at all co-located in the emissions in the two different wavelengths. According to the discussion above, the $\mathrm{H}_{3}^{+}$lifetime (a few seconds) combined with the estimated $\left(10-50 \mathrm{~m} \mathrm{~s}^{-1}\right)$ and the observed $\left(0.5-2.5 \mathrm{~km} \mathrm{~s}^{-1}\right)$ velocities in the co-rotation frame indicates that ions may be carried from a few tens of meters to several tens of kilometers and thus too small to be significant. For example, the increase in IR brightness between $185^{\circ}$ and $200^{\circ}$ in SIII longitude (Figure 4) cannot be explained by $\mathrm{H}_{3}^{+}$ion transport from the region where a localized peak of UV brightness is observed $\left(188^{\circ}-193^{\circ}\right.$ in SIII), since $10^{\circ}$ difference in SIII longitude at that latitude corresponds to approximately $8000 \mathrm{~km}$, much longer than the estimated transport distance above. As ion transport in the main auroral region cannot account for the large-scale discrepancies observed between the simultaneous UV and IR profiles, local heating seems to be a better candidate by affecting the gas temperature and thus the brightness of the $\mathrm{H}_{3}^{+}$emission. We propose that the difference in the brightness variations along the main emission in UV and IR are due to temporal temperature variations which might exist. These short-time scale variations, directly reflected by the UV emissions, are consistently delayed on the order of several hours in the IR thermal emissions. Finally, the energy of the electron population that excites the main emission (10 to hundreds keV [Gustin et al., 2004]) should also play a role in the discrepancies along the profiles measured at different wavelengths. The UV brightness profile is expected to increase near-linearly with energy, while the IR component is expected to drop as the precipitation hardens above about $10 \mathrm{keV}$ [Tao et al., 2011], leading to chemical loss of the low altitude $\mathrm{H}_{3}^{+}$.

\subsection{Polar Emission: UV-IR Morphological Comparison}

[25] The polar emissions in the UV do not always colocate with those in the IR. In particular, a bright polar patch observed in IR in the dawnside aurora does not have a UV counterpart. This patch is sampled in the latitudinal profile $\mathrm{f}$ and is evident in the IR brightness profile in Figure 3. The lack of agreement between the UV and IR polar emission is not surprising, given the fact that UV polar emissions at Jupiter consist of transient features that are observed to change rapidly within a few minutes (for example the swirl region [Grodent et al., 2003b] and polar flares [Waite et al. 2001; Bonfond et al., 2011]). The UV emissions are excited directly, the IR emissions are thermal emissions and last longer. A transient polar emission that disappeared in UV could be still present in IR. As it is estimated above, temperature variations would be reflected in the IR emissions with a time lag of several hours with respect to the UV and thus explaining the lack of agreement between the UV and IR polar emissions.

[26] Additionally, the brightness of the dark polar region in the UV is near zero, while in IR, the dark polar region emission is quite substantial. This points out to the much higher levels of $\mathrm{H}_{3}^{+}$emission in that region, compared to the $\mathrm{H}$ and $\mathrm{H}_{2}$. This can be explained by the fact that the precipitation is soft and thus favoring the IR/UV ratio. Furthermore, Joule heating (and thus temperature) is enhanced in this region. The corresponding magnetic field lines are quasi open and distant, exposed to the flow of the solar wind and thus will not rotate with the planet. The induced electric field mapped on the magnetic pole would drive currents in the ionosphere and cause additional Joule heating and increased temperature. The marginal energy flux deduced from the faint UV emission in the dark polar region suggests that auroral precipitation cannot explain the excess of IR emission [Grodent et al., 2003b; Stallard et al., 2003]. Another possibility could have been that $\mathrm{H}_{3}^{+}$is being transported from the auroral oval itself. However, as mentioned above, ion transport cannot account for the morphological differences over such large distances discussed here. Thus, we suggest that the enhanced IR emissions in the dark polar region are explained in terms of soft precipitation and most probably Joule heating.

\subsection{Equatorward Diffusion Emission: UV-IR Morphological Comparison}

[27] We find that the equatorward diffuse emission exists in both the UV and the IR but that it is not present in IR at all longitudes (e.g., it is absent along profile c). These emissions have been observed consistently in the UV confined to the region bounded by the main emission and the Io footprint, although the boundary between the main emission and the equatorward diffuse emission is not always obvious. Isolated UV auroral patches observed in that region have been related to magnetospheric injections and have been shown to have an IR counterpart [Bonfond et al., 2012]. Apart from these spot-like features, Radioti et al. [2009] concluded that part of the equatorward diffuse emission (arc-like structures) is related to electron scattering into the loss cone by whistler mode waves at the pitch angle distribution boundary, leading to electron precipitation in the ionosphere. The pitch 
RADIOTI ET AL.: UV-IR COMPARISON OF JOVIAN AURORA

Table 1. Relative Brightness Peak of Auroral Features (\% Main Emission)

\begin{tabular}{lcccccccc}
\hline Profile & UV-PE & IR-PE & UV-EDE & IR-EDE & UV-IFP & IR-IFP & UV-DPR & IR-DPR \\
\hline a & 30 & 45 & 65 & 60 & 35 & - & - & - \\
b & 140 & 100 & 240 & 60 & 380 & 45 & - & - \\
c & 55 & 170 & 70 & - & - & - & - & - \\
d & 80 & 160 & - & - & - & - & 20 & 80 \\
e & 45 & 150 & - & - & - & - & 10 & 90 \\
$\mathrm{f}$ & 40 & 70 & - & - & - & - & 5 & 55 \\
\hline
\end{tabular}

angle distribution boundary is regularly observed and it is considered to be stable in time [Tomás et al., 2004], thus explaining the simultaneity of the UV and IR components. The observations here point to co-location of the UV and IR equatorward diffuse emission, although we do not observe IR equatorward diffuse emission in all the latitudinal cuts in which they are observed in the UV. We suggest that the lack of diffuse equatorward emissions near SIII longitude $140^{\circ}-160^{\circ}$ is related to the maximum surface magnetic field in that region on the Northern Hemisphere [Connerney et al., 1998] and thus weaker electron precipitation. Additionally, there is a discrepancy with respect to the relative brightness compared with the main emission (e.g., along profile b, see Table 1). We propose that a possible explanation for the occasional absence of low brightness of IR in the equatorward diffuse emission relative to the main emission is related to the electron energy spectrum associated with this emission. Radioti et al. [2009] suggested that an electron population in the range of $15-300 \mathrm{keV}$ under the assumption of pitch angle diffusion could reproduce the observed UV equatorward diffuse emission. The high energy part of the electron distribution (100-300 keV) would precipitate below the homopause, so that the fraction of the $\mathrm{H}_{3}^{+}$column below the homopause would be rapidly destroyed.

\subsection{Io Footprint Emission: UV-IR Morphological Comparison}

[28] The comparison of the Io footprint in the UV and IR is more challenging. Figure 1 clearly demonstrates that the Io footprint and its tail have a UV and IR auroral counterpart. However, the IR footprint seems to be more diffuse than in the UV. The comparative profiles (Figure 2) show that the relative brightness of the Io footprint in the two wavelengths differs significantly in this pair of images. Here the brightness of the UV is up to 4 times larger than that of the main emission, while in the IR, it is remarkably dimmer than the main emission. The fact that the IR footprint looks more diffuse could be due to the point spread function at this particular time. If the observer's point spread function is large compared to the source spatial distribution, one should expect variations in the appearance of a spatially localized source. The differences in the relative to the main emission brightness of the UV and IR Io footprint could be also due to a fundamental discrepancies in the energy distribution of the incoming particles in this region of the auroral atmosphere as compared with the main emission. Studies of the Io footprint in the UV have shown that the altitude of the emission of the UV footprint is about $600-900 \mathrm{~km}$ [Bonfond et al., 2009; Bonfond, 2010]. The altitude of the IR Io footprint emissions has not been determined. Limb observations by Lystrup et al. [2008] have shown that IR auroral emissions in the southern main auroral oval extend to thousands of kilometers, but the peak altitude was ambiguous and limb observations of the Io footprint specifically have so far not been performed. The temperature in the region of the Io footprint is also unmeasured. However, at an altitude of $\sim 1000 \mathrm{~km}$, past observations suggest the $\mathrm{H}_{3}^{+}$ temperature may be as high as $1100 \mathrm{~K}$ [Lystrup et al., 2008]. Recent studies showed that the altitude of the UV tail emission is $900 \mathrm{~km}$ [Bonfond et al., 2009] and thus indicative of soft characteristic electron energies, implying the preferential excitation of the $\mathrm{H}_{3}^{+}$IR emission. The dimming of the Io tail in IR compared with UV found here and in previous studies (for example in Connerney and Satoh [2000]) could be explained in terms of the electron distribution. The Io footprint is caused by a very broad electron energy distribution extending from low energies of $0.5 \mathrm{keV}$ (responsible for weak emissions) and high energies beyond $20 \mathrm{keV}$ (leading to absorbed emissions). The strong and sudden energy input under the Io footprint could cause some upwelling bringing hydrocarbons to higher altitude (which would explain the UV color ratios) and would thus dim the IR emissions.

\section{Summary and Conclusions}

[29] In this work, we have discussed the brightness variations of several auroral features in UV and IR emission on the basis of simultaneous observations. Our analysis is based on relative brightness profiles as a function of angular distance measured from the morphological center of the main emission. Table 1 summarizes the relative brightness of the peak of the auroral features and Table 2 their co-location in UV and IR. This study demonstrates that (1) the UV and IR main emission are co-located, while the brightness profile along the main emission varies differently, and (2) the polar emissions are not always co-located in UV and IR, they are almost always dimmer than the main emission in UV and much brighter than the main emission in the IR. While the relative brightness of the dark polar region is near zero in UV, it is significantly brighter in IR. (3) The UV and IR equatorward diffuse emissions are co-located, and (4) the Io footprint and its tail are co-located in UV and IR, but they are significantly dimmer relative to the main emission in IR compared to UV in this pair of images.

Table 2. Co-location of Auroral Features

\begin{tabular}{|c|c|}
\hline Feature & Co-location in UV and IR \\
\hline Main emission & Co-located \\
\hline Main emission discontinuity & Exists in UV but not clear if present in IR \\
\hline Polar emission & Not always co-located \\
\hline Dark polar region & Co-located \\
\hline Equatorward emission & Co-located \\
\hline Io footprint & Co-located \\
\hline
\end{tabular}


[30] Three main factors may contribute to the observed morphological differences at the two wavelengths, namely the energy of the precipitating electrons, ion transport, and local heating. We estimate the $\mathrm{H}_{3}^{+}$ion transport distances and conclude that ion transport cannot account for the morphological differences between the UV and IR emissions. We suggest that neutral gas heating locally enhances the $\mathrm{H}_{3}^{+}$ emission with no UV counterpart. We estimate that local temperature variations are reflected in the IR emission with a time lag of several hours with respect to the UV. This time lag justifies the local time morphological discrepancies along the main emission in the IR and UV and explains the differences in the transient and short lived polar emissions in the two bandwidths. Additionally, we conclude that the enhanced IR emissions in the dark polar region could be explained mainly in terms of Joule heating. Finally, electron precipitation with energies above $10 \mathrm{keV}$ might lead to chemical loss of the low altitude $\mathrm{H}_{3}^{+}$ions, and thus contribute to the observed differences between the IR and UV emissions.

[31] Acknowledgments. This work is based on observations with the STIS instrument on the Hubble Space Telescope and IR imagery obtained with the NSFCAM instrument at NASA's Infrared Telescope Facility (IRTF). The IR imagery was obtained by Connerney and Satoh as part of a long-term observing campaign and archived for community use by Makenzie Lystrup. The Institute for Astronomy at the University of Hawaii operates the IRTF for NASA. The research was supported by the Belgian Fund for Scientific Research (FNRS) and the PRODEX Program managed by the European Space Agency in collaboration with the Belgian Federal Science Policy Office. A.R. is funded by the Belgian Fund for Scientific Research (FNRS). Robert Lysak thanks Stanley Cowley and another reviewer for their assistance in evaluating this paper.

\section{References}

Achilleos, N., S. Miller, R. Prang, G. Millward, and M. K. Dougherty (2001), A dynamical model of, Jupiter's auroral electrojet, New J. Phys., 3, 1-20, doi:10.1088/1367-2630/3/1/303.

Ballester, G. E., et al. (1996), Time-resolved observations of Jupiter's farultraviolet aurora, Science, 274, 409-412.

Bonfond, B. (2010), The 3-D extent of the, Io UV footprint on Jupiter, $J$. Geophys. Res., 115, A09217, doi:10.1029/2010JA015475.

Bonfond, B., D. Grodent, J.-C. Gérard, A. Radioti, V. Dols, P. A. Delamere, and J. T. Clarke (2009), The Io UV footprint: Location, inter-spot distances and tail vertical extent, J. Geophys. Res., 114, A07224, doi: 10.1029/2009JA014312.

Bonfond, B., M. F. Vogt, J.-C. Gérard, D. Grodent, A. Radioti, and V. Coumans (2011), Quasi-periodic polar flares at Jupiter: A signature of pulsed dayside reconnections? Geophys. Res. Lett., 38, L02104, doi: 10.1029/2010GL045981.

Bonfond, B. (2012), When moons create aurora: The satellite footprints on giant planets in auroral phenomenology and magnetospheric processes: Earth and other planets, in Geophys. Monogr. Ser, edited by Keiling, A., et al., pp. 133-140, vol. 197, AGU, Washington, D. C., doi: $10.1029 / 2011$ GM001169, (to appear in print).

Bonfond, B., D. Grodent, J.-C. Gérard, T. Stallard, J. T. Clarke, M. Yoneda A. Radioti, and J. Gustin (2012), Auroral evidence of Io's control over the magnetosphere of Jupiter, Geophys. Res. Lett., 39, doi: 10.1029/2011GL050253.

Bougher, S. W., J. H. Waite Jr., T. Majeed, and G. R. Gladstone (2005), Jupiter Thermospheric General Circulation Model (JTGCM): Global structure and dynamics driven by auroral and Joule heating, J. Geophys. Res., 110, E04008, doi:10.1029/2003JE002230.

Bunce, E. J., S. W. H. Cowley, and T. K. Yeoman (2004), Jovian cusp processes: Implications for the polar aurora, J. Geophys. Res., 109, A09S13, doi:10.1029/2003JA010280.

Clarke, J. T., et al. (1996), Far-ultraviolet imaging of Jupiter's aurora and the Io "footprint", Science, 274, 404-409.

Clarke, J. T., et al. (2002), Ultraviolet emissions from the magnetic footprints of Io, Ganymede and Europa on Jupiter, Nature, 415, 997-1000.
Clarke, J. T., D. Grodent, S. W. H. Cowley, E. J. Bunce, P. Zarka, J. E. P. Connerney, and T. Satoh (2004), Jupiter's aurora, in Jupiter. The Planet, Satellites and Magnetosphere, pp. 639-670, Cambridge Univ. Press, Cambridge, U. K.

Connerney, J. E. P., and T. Satoh (2000), The $\mathrm{H}_{3}^{+}$ion: A remote diagnostic of the Jovian magnetosphere, Phil. Trans. R. Soc. Lond. A, 358, 2471-2483.

Connerney, J. E. P., R. Baron, T. Satoh, and T. Owen (1993), Images of excited $\mathrm{H}_{3}^{+}$at the foot of the Io flux tube in Jupiter's atmosphere, Science, 262, 1035-1038, doi:10.1126/science.262.5136.1035.

Connerney, J. E. P., M. H. Acuna, N. F. Ness, and T. Satoh (1998), New models of Jupiter's magnetic field constrained by the Io flux tube footprint, J. Geophys. Res., 103, 11929-11939.

Cowley, S. W. H., and E. J. Bunce (2001), Origin of the main auroral oval in Jupiter's coupled magnetosphere-ionosphere system, Planet. Space Sci., 49, 1067-1088.

Cowley, S. W. H., E. J. Bunce, T. S. Stallard, and S. Miller (2003), Jupiter's polar ionospheric flows: Theoretical interpretation, Geophys. Res. Lett., 30(5), 1220, doi:10.1029/2002GL016030.

Gérard, J.-C., V. Dols, R. Prange, and F. Paresce (1994), The morphology of the north Jovian ultraviolet aurora observed with the Hubble Space Telescope, Planet. Space Sci., 42, 905-917.

Gérard, J.-C., J. Gustin, D. Grodent, J. T. Clarke, and A. Grard (2003), Spectral observations of transient features in the, FUV Jovian polar aurora, $J$. Geophys. Res., 108(A8), 1319, doi:10.1029/2003JA009901.

Grodent, D., J. H. Waite Jr., and J.-C. Gérard (2001), A self-consistent model of the, Jovian auroral thermal structure, J. Geophys. Res., 106(A7), 12933-12952, doi:10.1029/2000JA900129.

Grodent, D., J. T. Clarke, J. Kim, J. H. Waite Jr., and S. W. H. Cowley (2003a), Jupiter's main auroral oval observed with HST-STIS, $J$. Geophys. Res. Lett., 180, 1389, doi:10.1029/20003JA009921.

Grodent, D., J. T. Clarke, J. H. Waite Jr., S. W. H. Cowley, J.-C. Gérard, and J. Kim (2003b), Jupiter's polar auroral emissions, J. Geophys. Res., 108, 1366, doi:10.1029/20003JA010017.

Grodent, D., J.-C. Gérard, J. T. Clarke, G. R. Gladstone, and J. H. Waite Jr. (2004), A possible auroral signature of magnetotail reconnection process on Jupiter, J. Geophys. Res., 109, A05201, doi:10.1029/ 2003JA010341.

Grodent, D., J.-C. Gérard, J. Gustin, B. H. Mauk, J. E. P. Connerney, and J. T. Clarke (2006), Europa's FUV auroral tail on Jupiter, Geophys. Res., Lett., 33, L06201, doi:10.1029/2005GL025487.

Grodent, D., B. Bonfond, J.-C. Gérard, A. Radioti, J. Gustin, J. T. Clarke, J. Nichols, and J. E. P. Connerney (2008), Auroral evidence of a localized magnetic anomaly in Jupiter's northern hemisphere, J. Geophys. Res., 113, A09201, doi:10.1029/2008JA013185.

Grodent, D., B. Bonfond, A. Radioti, J.-C. Gérard, X. Jia, J. D. Nichols, and J. T. Clarke (2009), Auroral footprint of, Ganymede, J. Geophys. Res., 114, A07212, doi:10.1029/2009JA014289.

Gurnett, D. A., and C. K. Goertz (1981), Multiple Alfven wave reflections excited by Io: Origin of the Jovian decametric arcs, J. Geophys. Res., 86, $717-722$.

Gustin, J., J.-C. Gérard, D. Grodent, S. W. H. Cowley, J. T. Clarke, and A. Grad (2004), Energy-flux relationship in the FUV Jovian aurora deduced from HST-STIS spectral observations, J. Geophys. Res., 109, A10205.

Gustin, J., B. Bertrand, D. Grodent, and J.-C. Gérard (2012), Conversion from HST ACS and STIS auroral counts into brightness, precipitated power, and radiated power for $\mathrm{H} 2$ giant planets, J. Geophys. Res., 117, A07316, doi:10.1029/2012JA017607.

Hill, T. W. (2001), The Jovian auroral oval, J. Geophys. Res., 106, 8101-8107.

Hill, T. W., and V. M. Vasyliûnas (2002), Jovian auroral signature of Io's corotational wake, J. Geophys. Res., 107 (A12), 1464, doi: 10.1029/2002JA009514

Kao, L., T. Oka, S. Miller, and J. Tennyson (1991), A table of astronomically important ro-vibrational transitions for the $\mathrm{H}_{3}^{+}$molecular ion, Astroph. J. Supp. Series, 77, 317-329, doi:10.1086/191606.

Lystrup, M. B., S. Miller, R. Dello N., R. J. Vervack, and T. Stallard (2008), First vertical ion density profile in Jupiter's upper atmosphere: Direct observations using the Keck II Telescope, ApJ, 677, 790-797, doi: 10.1086/529509.

Mauk, B. H., J. T. Clarke, D. Grodent, J. H. Waite Jr., C. P. Paranicas, and D. J. Williams (2002), Transient aurora on Jupiter from injections of magnetospheric electrons, Nature, 415, 1003-1005.

Neubauer, F. M. (1980), Non-linear Alfven wave current system at Io: Theory, J. Geophys. Res., 85, 1171-1178.

Nichols, J. D., J. T. Clarke, J. C. Gérard, and D. Grodent (2009a), Observations of Jovian polar auroral filaments, Geophys. Res. Lett., 36, L08101, doi:10.1029/2009GL037578. 


\section{RADIOTI ET AL.: UV-IR COMPARISON OF JOVIAN AURORA}

Nichols, J. D., J. T. Clarke, J. C. Gérard, D. Grodent, and K. C. Hansen (2009b), Variation of different components of Jupiterís auroral emission, J. Geophys. Res., 114, A06210, doi:10.1029/2009JA014051.

Radioti, A., J.-C. Gérard, D. Grodent, B. Bonfond, N. Krupp, and J. Woch (2008a), Discontinuity in Jupiter's mail auroral oval, J. Geophys. Res., 113, A01215, doi:10.1029/2007JA012610.

Radioti, A., D. Grodent, J.-C. Gérard, B. Bonfond, and J. T. Clarke (2008b), Auroral polar dawn spots: Signatures of internally driven reconnection processes at Jupiter's magnetotail, Geophys. Res. Lett., 35, L03104, doi: 10.1029/2007GL032460.

Radioti, A., A. T. Tomás, D. Grodent, J.-C. Gérard, J. Gustin, B. Bonfond N. Krupp, J. Woch, and J. D. Menietti (2009), Equatorward diffuse auroral emissions at Jupiter: Simultaneous HST and Galileo observations, Geophys. Res. Lett., 36, L07101, doi:10.1029/2009GL037857.

Radioti, A., D. Grodent, J.-C. Gérard, M. F. Vogt, M. Lystrup, and B. Bonfond (2011), J. Geophys. Res., 116, A03221, doi:10.1029/ 2010JA016200.

Raynaud, E., E. Lellouch, J.-P. Maillard, G. R. Gladstone, J. H. Waite Jr., B. Bézard, P. Drossart, and T. Fouchet (2004), Spectro-imaging observations of Jupiter's $2-\mu \mathrm{m}$ auroral emission. I $H_{3}^{+}$distribution and temperature, Icarus, 171, 133-152, doi:10.1016/j.icarus.2004. 04.020 .

Rego, D., et al. (1999), Supersonic winds in Jupiter's aurorae, Nature, 399 , 121-124, doi:10.1038/20121.

Satoh, T., and J. E. P. Connerney (1999), Jupiter's $H_{3}^{+}$emissions viewed in corrected jovimagnetic coordinates, Icarus, 141, 236-252, doi: 10.1006/icar.1999.6173
Shure, M., et al. (1994), A powerful new infrared array camera for the NASA infrared telescope facility, Experimental Astronomy, 3 (1-4), 239-242, doi: 10.1007/BF00430170.

Stallard, T., S. Miller, G. Millward, and R. D. Joseph (2001), On the dynamics of the Jovian ionosphere and thermosphere. I. The measurement of ion winds, Icarus, 154, 475-491, doi:10.1006/icar.2001.6681.

Stallard, T., S. Miller, G. Millward, and R. D. Joseph (2002), On the dynamics of the Jovian ionosphere and thermosphere. II. The measurement of $\mathrm{H}_{3}^{+}$vibrational temperature, column density, and total emission, Icarus, 156, 498-514, doi:10.1006/icar.2001.6793.

Stallard, T. S., S. Miller, S. W. H. Cowley, and E. J. Bunce (2003), Jupiter's polar ionospheric flows: Measured intensity and velocity variations poleward of the main auroral oval, Geophys. Res. Lett., 30(5), 1221, doi: 10.1029/2002GL016031

Tao, C., S. V. Badman, and M. Fujimoto (2011), UV and IR auroral emission model for the outer planets: Jupiter and Saturn comparison, Icarus, 213,2, 581-592, doi:10.1016/j.icarus.2011.04.001.

Tomás, A. J. Woch, N. Krupp, A. Lagg, K.-H. Glassmeier, M. K. Dougherty, and P. Hanlon (2004), Changes of the energetic particles characteristics in the inner part of the Jovian magnetosphere: A topological study, Planet. Space Sci., 52, 491-498, doi:10.1016/ j.pss.2003.06.011

Vasavada, A. R., A. H. Bouchez, A. P. Ingersoll, B. Little, and C. D. Anger (1999), Jupiter's visible aurora and Io footprint, J. Geophys. Res., 104(E11), 27133-27142, doi:10.1029/1999JE001055.

Waite, H., et al. (2001), An auroral flare at Jupiter, Nature, 410, 6830, $787-789$. 